\title{
Ansiedade em Meio Clínico: Construção de uma Escala para Estudantes de Medicina
}

\author{
Anxiety in Clinical Settings: Constructing a Scale for Medical Students
}

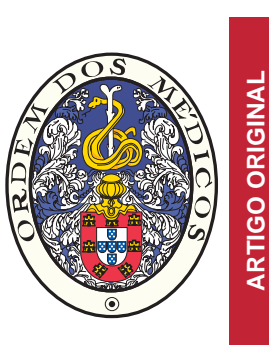

Joana Ramos RODRIGUES ${ }^{1}$, Miguel CASTELO-BRANCO ${ }^{1}$, Henrique PEREIRA², Rosa Marina AFONSO ${ }^{3}$

Acta Med Port 2014 Nov-Dec;27(6):731-736

RESUMO

Introdução: A Escala de Ansiedade em Meio Clínico foi construída para avaliar o nível de ansiedade dos estudantes de Medicina quando expostos ao meio clínico e identificar quais as situações mais propensas a desencadear maior nível de ansiedade nesse contexto.

Material e Métodos: Este instrumento é constituído por doze itens e foi construído tendo por base a revisão da literatura sobre a temática da ansiedade em meio clínico e os dados provenientes de um focus group realizado com estudantes entre o $1^{\circ}$ e o $5^{\circ}$ ano do curso de Medicina da Universidade da Beira Interior $(n=10)$. As suas propriedades psicométricas foram testadas mediante um estudo com 557 alunos do curso de Medicina de quatro universidades portuguesas, entre o $1^{\circ} \mathrm{e} \circ 6^{\circ}$ ano.

Resultados: A validação da Escala de Ansiedade em Meio Clínico contemplou a análise de três parâmetros principais: sensibilidade, fiabilidade e análise da estrutura fatorial, sendo que a última resultou na obtenção de quatro factores: 'Incómodo perante procedimentos invasivos', 'Ansiedade face ao doente', 'Ansiedade face ao desempenho' e 'Ansiedade face à dimensão humana' com 70,6\% da variância explicada.

Discussão: A Escala de Ansiedade em Meio Clínico total demonstra uma boa coerência interna (Alpha de Cronbach $=0,84)$ e boa capacidade de discriminação de sujeitos, apresentando-se, assim, como instrumento consistente e fiável para a avaliação de ansiedade nos estudantes de Medicina quando expostos ao meio clínico.

Conclusão: A Escala de Ansiedade em Meio Clínico permite avaliar a ansiedade nos estudantes de Medicina quando expostos ao meio clínico e poderá vir a ser útil na delineação de estratégias de ensino para a preparação dos futuros médicos.

Palavras-chave: Ansiedade; Escalas; Ensino Médico Pré-Graduado; Estudantes de Medicina; Stress Psicológico.

\section{ABSTRACT}

Introduction: The Scale of Anxiety in Clinical Setting (SACS) was constructed to assess the level of anxiety of medical students when exposed to the clinical setting and also to identify what situations are more likely to trigger higher levels of anxiety in that context.

Material and Methods: This instrument consists of 12 items and was constructed on the basis of the review of the literature on the topic of anxiety in clinical setting, and data from a focus group conducted with students between the 1st and the 5th year of the Medical course at University of Beira Interior $(n=10)$. The Scale of Anxiety in Clinical Setting psychometric properties were tested through a study with 557 medical students of four Portuguese universities, between the $1^{\text {st }}$ and the $6^{\text {th }}$ year.

Results: The Scale of Anxiety in Clinical Setting validation included the analysis of three key parameters: sensitivity, reliability and factorial validity, the last resulting in four factors: 'displeasure before invasive procedures', 'anxiety toward the sick', 'anxiety about performance' and 'anxiety toward the human dimension' with $70.6 \%$ of the variance explained.

Discussion: Total Scale of Anxiety in Clinical Setting shows a good internal consistency (Cronbach's Alpha $=0.84$ ) and a good discriminatory ability, presenting as a consistent and reliable instrument for the assessment of anxiety in medical students when exposed to the clinical setting.

Conclusion: The Scale of Anxiety in Clinical Setting evaluates the anxiety in medical students when exposed to the clinical setting and may be useful in the delineation of teaching strategies for the preparation of future doctors.

Keywords: Anxiety; Education, Medical, Undergraduate; Students, Medical; Stress, Physiological; Questionnaires.

\section{INTRODUÇÃo}

O curso de Medicina é um dos cursos mais exigentes do Ensino Superior Português e, se por um lado, permite o acesso a uma significativa realização profissional, por outro lado, pressupõe anos de trabalho e uma dedicação exclusiva que se inicia logo a partir dos primeiros anos do percurso formativo. Os estudantes de Medicina que apresentam, frequentemente, indicadores associados a elevados níveis de morbilidade psicológica na qual se inclui a ansiedade, ${ }^{1-3}$ têm sido alvo de várias investigações para avaliar os níveis de bem-estar e saúde, assim como as circunstâncias que possam estar relacionadas com esta maior vulnerabilidade à patologia do foro psiquiátrico.

A palavra ansiedade deriva do latim anxietas 4 e é o termo utilizado para expressar um sentimento de apreensão, preocupação, inquietação ou temor. ${ }^{5}$ Apesar da sua conotação habitualmente negativa, a ansiedade pode, contudo, desempenhar um papel protetor ou adaptativo. A ansiedade torna-se patológica quando excessiva, interferindo com o funcionamento adaptativo do sujeito..$^{5,6} \mathrm{~A}$ ansiedade pode ser desencadeada, entre outros factores, por situações de stress, sendo uma consequência da dificuldade em gerir os recursos disponíveis para manter o equilíbrio.

\footnotetext{
1. Faculdade de Ciências da Saúde. Universidade da Beira Interior. Covilhã. Portugal.

2. Departamento de Psicologia e Educação. Unidade de Investigação em Psicologia e Saúde. Universidade da Beira Interior. Covilhã. Portugal.

3. Departamento de Psicologia e Educação. Unidade de Investigação e Formação sobre Adultos e Idosos. Universidade da Beira Interior. Covilhã. Portugal. Recebido: 30 de Maio de 2014 - Aceite: 28 de Outubro de 2014 | Copyright $\odot$ Ordem dos Médicos 2014
} 
A carreira médica e, especificamente, o curso de Medicina, têm sido tradicionalmente associados a níveis elevados de stress e ansiedade, ${ }^{7,8-11}$ o que é consistente com o facto das profissões da área da Saúde ocuparem o terceiro lugar da classificação de ocupações com maior intensidade de stress. ${ }^{12} \mathrm{~A}$ psicopatologia entre médicos apresenta uma prevalência superior à da população em geral, ${ }^{9,11} \mathrm{com}$ até $52,4 \%$ destes profissionais em burnout, ${ }^{9}$ sendo que também os alunos de Medicina parecem apresentar uma prevalência elevada de morbilidade psicológica, nomeadamente depressão, suicídio e uso de drogas. . $^{8,12,13}$

Para além da admissão ao curso de Medicina constituir um processo altamente exigente, a trajetória académica do futuro médico implica um percurso formativo associado a importantes fatores stressantes, tais como a pressão para assimilar grande quantidade de novas informações, a falta de tempo para atividades sociais, ${ }^{7}$ o primeiro contacto com o sofrimento do doente e com a morte, entre outros. O início do envolvimento em meio clínico pode associar-se a um aumento dos sintomas obsessivos e de ansiedade, bem como a níveis significativos de stress. ${ }^{8,14}$ Assim, constata-se que até $21 \%$ dos alunos do curso de Medicina relatam sentir-se ansiosos. ${ }^{12}$

As perturbações de ansiedade durante a adolescência constituem um fator de risco para o surgimento posterior de ansiedade, depressão e comportamentos aditivos ${ }^{15}$. A carreira médica associa-se também a elevados níveis de stress laboral e morbilidade psicológica, com repercussões negativas na saúde e no bem-estar do próprio médico, com possíveis implicações negativas na assistência ao doente. Assim, é importante identificar e alterar situações associadas a maiores níveis de stress e ansiedade nos estudantes de Medicina, por forma a garantir menor morbilidade psicológica durante a sua formação e futuro percurso profissional.

Os objetivos principais desta investigação são: (1) construir uma escala de avaliação da ansiedade em meio

Tabela 1 - Características sociodemográficas da amostra $(n=557)$

\begin{tabular}{|lcc|}
\hline Idade & $\boldsymbol{n}$ & $\%$ \\
\hline$<19$ & 149 & 26,8 \\
$20-21$ & 73 & 13,1 \\
$22-23$ & 153 & 27,5 \\
$24-25$ & 143 & 25,6 \\
$\geq 26$ & 39 & 7,0 \\
\hline Sexo & & \\
\hline Feminino & 388 & 69,7 \\
Masculino & 169 & 30,3 \\
\hline Estudante na cidade de residência & & \\
\hline Sim & 264 & 47,4 \\
Não & 293 & 52,6 \\
\hline Com quem reside & & \\
\hline Sozinho & 53 & 9,5 \\
Com pais/familiares & 251 & 45,1 \\
Com amigos/colegas & 210 & 37,7 \\
Outro & 43 & 7,7 \\
\hline
\end{tabular}

clínico para estudantes de Medicina; (2) avaliar os níveis de ansiedade em estudantes de Medicina quando expostos ao meio clínico; e (3) identificar as principais situações indutoras de ansiedade em contexto clínico.

\section{MATERIAL E MÉTODOS}

A construção da Escala de Avaliação da Ansiedade em Meio Clínico (EAMC) (Apêndice 1) implicou, numa primeira fase, pesquisa bibliográfica acerca das principais fontes de ansiedade em meio clínico e, posteriormente, a recolha de dados a partir de um focus group realizado com alunos do curso de Medicina (Tabelas 1, 2 e 3). Este consistiu na dinamização de um grupo de dez alunos da Faculdade de Ciências da Saúde entre o $1^{\circ}$ e $5^{\circ}$ anos do curso de Medicina da Universidade da Beira Interior, que pretendeu identificar as principais situações vivenciadas durante os estágios em meio clínico associadas a maiores níveis de ansiedade. O focus group decorreu numa sala de aula da Faculdade de Ciências da Saúde e teve a duração de cerca de uma hora. A participação dos alunos foi voluntária.

De acordo com a revisão bibliográfica efetuada, as situações associadas a maiores níveis de ansiedade foram: contacto com o sofrimento e a morte, contacto com os pacientes, dissecções de cadáveres (autópsias) e visualização de sangue. Os resultados do focus group identificaram situações relacionadas com a inflicção de dor aos doentes durante um procedimento, contacto com os doentes mais fragilizados, comunicação com o doente, atividades em meio clínico sob supervisão de um tutor, colegas ou outros, autópsias, contacto com a morte, visualização de sangue e discrepância entre os protocolos de diferentes técnicas lecionadas na faculdade e a sua aplicação em meio clínico. Desta forma, construíram-se 13 itens. De salientar que foi inserido um item relativo ao desmaio (item 13) apesar de não ter sido encontrado durante a revisão bibliográfica ou referido durante o focus group, devido ao facto de ser uma manifestação 'tradicionalmente' associada a algumas situa-

Tabela 2 - Características académicas da amostra $(n=557)$

\begin{tabular}{lcc}
\hline Estabelecimento de Ensino & $\boldsymbol{n}$ & $\%$ \\
\hline U. do Algarve & 35 & 6,2 \\
U. da Beira Interior & 135 & 24,3 \\
U. de Coimbra & 2 & 0,4 \\
U. de Lisboa & 381 & 68,3 \\
Outras Universidades * & 4 & 0,8 \\
\hline Ano de Curso & & \\
\hline $1^{\circ}$ & 126 & 22,6 \\
$2^{\circ}$ & 89 & 16,0 \\
$3^{\circ}$ & 78 & 14,0 \\
$4^{\circ}$ & 93 & 16,7 \\
$5^{\circ}$ & 84 & 15,1 \\
$6^{\circ}$ & 87 & 15,6 \\
\hline
\end{tabular}

*Uma vez que não se obteve colaboração formal de outras Universidades, é muito provável que a seleção da opção correspondente a estes estabelecimentos de ensino se tenha devido a enganos durante o preenchimento do questionário. 
Tabela 3 - Autoavaliação do desempenho académico e satisfação com o curso por parte dos estudantes do Ensino Superior $(n=557)$

\begin{tabular}{lcc}
\hline Autoavaliação do desempenho académico & $\boldsymbol{n}$ & $\%$ \\
\hline Péssimo & 0 & 0 \\
Mau & 8 & 1,4 \\
Razoável & 210 & 37,7 \\
Bom & 320 & 57,5 \\
Excelente & 19 & 3,4 \\
Satisfação com o curso & & \\
Péssimo & 0 & 0 \\
Mau & 15 & 2,7 \\
Razoável & 100 & 18,0 \\
Bom & 294 & 52,7 \\
Excelente & 148 & 26,6 \\
\hline
\end{tabular}

ções atrás mencionadas. Os itens foram avaliados através de uma escala do tipo Likert, com quatro posições para a indicação da intensidade da ansiedade associada a cada questão/situação. Quanto maior a cotação atribuída a um item, maior ansiedade associada ao mesmo. Não existem itens cotados inversamente.

Para efeitos da aplicação do questionário, as diferentes Universidades foram contactadas previamente por via telefónica, explicando os objetivos do estudo e solicitando a colaboração de todos os alunos da instituição. A decisão da aplicação ou não do questionário ficou a cargo das entidades responsáveis. O questionário esteve disponível numa plataforma online construída no âmbito deste estudo, entre 14 de outubro e 14 de novembro de 2013.

Após a construção da escala, a validação psicométrica foi realizada através do estudo das características psicométricas (sensibilidade, fiabilidade e análise da estrutura factorial) dos resultados da aplicação da EAMC a alunos de Medicina de diversas universidades portuguesas.

Os resultados obtidos foram analisados com o programa Statistical Package for Social Sciences, versão 21 para Microsoft Windows.

\section{RESULTADOS}

\section{Validação da EAMC}

Relativamente à EAMC, tratando-se do processo de construção do instrumento, efetuou-se a análise da sensibilidade através das medidas de assimetria (sk) e curtose (ku). Uma vez que um dos itens apresentava um valor de curtose $=14,344$ (> 3) (item 13), este foi removido. ${ }^{16} \mathrm{~A}$ coerência interna foi então avaliada após a exclusão do item referido, através do Alpha de Cronbach. Seguidamente, realizou-se a análise fatorial precedida do teste de Kaiser-Meyer-Olkin (KMO) e a Rotação Varimax foi utilizada para agrupar a totalidade dos itens em diferentes fatores.

Obtiveram-se quatro dimensões com posterior estimação da fiabilidade pelo Alpha de Cronbach para cada uma das dimensões (Tabela 4).

Dado o número elevado de participantes $(n=557)$ assumiu-se, de acordo com o Teorema do Limite Central, que a distribuição da mesma é normal ${ }^{16}$, tendo-se ainda assim, explorado e confirmado a normalidade da distribuição através do teste de Kolmogorov-Smirnov.

\section{Sensibilidade}

A sensibilidade dos itens foi avaliada pelas medidas de assimetria ( $s k$ ) e curtose $(k u)$. Os dados obtidos remetem para um instrumento com boa capacidade para discriminar os sujeitos, com a exceção do item 13, que apresentou um valor de curtose $=14,34(>3)$, tendo sido removido. ${ }^{16} \mathrm{Op}$ tou-se por não excluir os itens 3, 5 e 7 apesar de apresentarem um valor de curtose $>3$, uma vez que a sua contribuição teórica foi considerada pertinente para a avaliação do construto. Além disso, a exclusão destes itens prejudicaria a coerência interna do instrumento, reduzindo o Alpha de Cronbach de 0,84 para 0,82 (Tabela 5).

\section{Fiabilidade}

A coerência interna foi avaliada após a exclusão do item 13, tendo-se obtido um Alpha de Cronbach de 0,84 , o que corresponde a uma classificação qualitativa boa. ${ }^{17}$

\section{Análise da estrutura fatorial}

Para a validação da adequação da amostra com recurso ao método de análise fatorial exploratória com extracção dos componentes principais, calculou-se a medida de $\mathrm{KMO}$, obtendo-se um valor de $0,81(\geq 0,5)$, considerado muito bom. ${ }^{16}$

Dos quatro fatores obtidos, o primeiro explica $37,6 \%$ da variância dos resultados e satura em quatro itens, tendo sido designado por 'Incómodo perante procedimentos invasivos'. O segundo fator, 'Ansiedade face ao doente', explica $14,9 \%$ da variância dos resultados e satura em três itens. 'Ansiedade face ao desempenho' é o terceiro fator,

Tabela 4 - Mediana, medidas de assimetria (sk) e curtose ( $k u)$, mínimo e máximo para os 13 itens da $\operatorname{EAMC~}(n=557)$

\begin{tabular}{cccccc}
\hline Item & Me & $\boldsymbol{S k}$ & $\boldsymbol{K u}$ & Min & Max \\
\hline 1 & 2 & 0,64 & 0,76 & 1 & 4 \\
2 & 1 & 1,10 & 0,52 & 1 & 4 \\
3 & 1 & 2,96 & $\mathbf{9 , 7 0}$ & 1 & 4 \\
4 & 2 & 0,42 & $-0,18$ & 1 & 4 \\
5 & 1 & 2,28 & $\mathbf{4 , 6 5}$ & 1 & 4 \\
6 & 2 & 0,48 & $-0,22$ & 1 & 4 \\
7 & 1 & 1,86 & $\mathbf{3 , 2 9}$ & 1 & 4 \\
8 & 1 & 1,21 & 0,94 & 1 & 4 \\
9 & 2 & 0,53 & $-0,49$ & 1 & 4 \\
10 & 1 & 0,97 & 0,14 & 1 & 4 \\
11 & 2 & 0,45 & $-0,54$ & 1 & 4 \\
12 & 2 & 0,12 & $-0,81$ & 1 & 4 \\
13 & 1 & 3,64 & $\mathbf{1 4 , 3 4}$ & 1 & 4 \\
\hline
\end{tabular}


Tabela 5 - Pesos fatoriais de cada item nos 4 fatores retidos, coerência interna (Alpha de Cronbach), eigenvalues e \% da variância explicada, após uma AVE com extração de fatores pelo método das componentes principais, seguida de uma Rotação Varimax

\begin{tabular}{|c|c|c|c|c|}
\hline \multirow[b]{2}{*}{ Itens } & \multicolumn{4}{|c|}{ Fatores } \\
\hline & $\begin{array}{l}\text { Incómodo perante } \\
\text { procedimentos invasivos }\end{array}$ & $\begin{array}{l}\text { Ansiedade face ao } \\
\text { doente }\end{array}$ & $\begin{array}{l}\text { Ansiedade face ao } \\
\text { desempenho }\end{array}$ & $\begin{array}{l}\text { Ansiedade face à } \\
\text { dimensão humana }\end{array}$ \\
\hline 2 & 0,80 & & & \\
\hline 3 & 0,78 & & & \\
\hline 5 & 0,83 & & & \\
\hline 10 & 0,73 & & & \\
\hline 1 & & 0,71 & & \\
\hline 4 & & 0,77 & & \\
\hline 12 & & 0,72 & & \\
\hline 6 & & & 0,81 & \\
\hline 9 & & & 0,84 & \\
\hline 11 & & & 0,60 & \\
\hline 7 & & & & 0,61 \\
\hline 8 & & & & 0,74 \\
\hline Alpha & 0,80 & 0,71 & 0,70 & 0,52 \\
\hline Eigenvalue & 4,51 & 1,79 & 1,11 & 1,05 \\
\hline Variância explicada & $37,6 \%$ & $14,9 \%$ & $9,3 \%$ & $8,8 \%$ \\
\hline
\end{tabular}

explicando 9,3\% da variância dos resultados e saturando em três itens. Por último, o quarto fator, 'Ansiedade face à dimensão humana', explica $8,8 \%$ da variância dos resultados e satura em dois itens.

Os Alpha de Cronbach de todos os fatores são considerados bons ou satisfatórios, à exceção do fator 'Ansiedade face à dimensão humana', que apresenta um valor de 0,52 , considerado mau. ${ }^{17}$

\section{Resultados da EAMC}

Relativamente aos níveis de ansiedade em meio clínico, a pontuação mínima obtida foi de 12,00 e a máxima de 37,00 . A média foi de $20,72(D P=5,43)$ e a mediana observada 20,00 , enquanto que a mediana teórica (ponto de corte teoricamente esperado) de 30,00. Assim, tendo como referência este último valor, verificou-se que $91 \%$ dos estudantes de Medicina apresentaram baixos níveis de ansiedade em meio clínico. A 'possibilidade de infligir dor ou dano ao doente durante diferentes procedimentos' é o item que se associa a níveis de ansiedade em contexto clínico mais elevados, com uma média de $2,42(D P=0,92)$. Pelo contrário, o 'incómodo ao ver sangue' foi o parâmetro associado a menor ansiedade $(\mathrm{M}=1,21 ; D P=0,52)$. $\mathrm{O}$ fator relacionado com a 'ansiedade face ao doente', englobando os itens 2, 3, 5 e 10, foi o que apresentou maiores níveis de ansiedade em meio clínico, com uma média de 6,38 ( $D P=$ $1,91)$, enquanto que a 'ansiedade face à dimensão humana' apresentou uma média de 2,82 ( $D P=1,02)$ (Tabela 6).

Relativamente à perceção dos estudantes acerca das medidas dos estabelecimentos de ensino, constatou-se que $55,3 \%$ dos estudantes de Medicina consideraram que a sua faculdade não implementava atividades ou outras medidas que contribuíssem para a redução dos níveis de ansiedade em meio clínico. No que concerne à promoção de alguma forma de apoio relativamente a situações que pudessem desencadear maior grau de ansiedade aquando do envolvimento em meio clínico, 91\% dos estudantes de Medicina inquiridos, consideraram ser pertinente.

\section{DISCUSSÃO}

Durante o tratamento estatístico dos dados, verificou-se que quatro dos 13 itens (3, 5, 7 e 13) da escala apresentavam valores de curtose $>3$. Ainda assim, optou-se por não excluir os itens 3, 5 e 7, uma vez que a sua contribuição teórica é pertinente para a avaliação do construto e a sua exclusão se refletiu numa redução, ainda que ligeira, do Alpha de Cronbach calculado para a EAMC. O fator 'ansiedade face à dimensão humana' foi mantido apesar de apresentar um valor baixo de coerência interna $(\alpha=0,52)$, uma vez que tanto a dissecção de cadáveres como a comunicação com o doente, itens englobados neste componente, foram situações bastante enfatizadas durante a realização do focus group. Considerou-se pois que seria importante conservar este fator. Assim, tendo como referência a mediana teórica da escala supracitada, $91 \%$ dos estudantes de Medicina apresentam níveis baixos de ansiedade em meio clínico. Em concordância com outros estudos, ${ }^{8,12}$ os itens associados ao sofrimento do paciente foram os mais frequentemente indicados como causadores de ansiedade.

O facto de não ter sido realizada uma validação por 
Tabela 6 - Média, mediana, valor mínimo e máximo dos resultados obtidos pelos estudantes de Medicina nos diferentes fatores da EAMC $(n=557)$

\begin{tabular}{|c|c|c|c|c|c|}
\hline & & $\begin{array}{c}\text { Incómodo perante } \\
\text { procedimentos invasivos }\end{array}$ & $\begin{array}{l}\text { Ansiedade face ao } \\
\text { doente }\end{array}$ & $\begin{array}{l}\text { Ansiedade face ao } \\
\text { desempenho }\end{array}$ & $\begin{array}{l}\text { Ansiedade face à } \\
\text { dimensão humana }\end{array}$ \\
\hline \multirow{2}{*}{$N$} & Válidos & 339 & 367 & 352 & 221 \\
\hline & Missing & 218 & 190 & 205 & 336 \\
\hline Média & & 5,74 & 6,38 & 6,14 & 2,82 \\
\hline Mediana & & 5,00 & 6,00 & 6,00 & 3,00 \\
\hline DP & & 2,19 & 1,91 & 2,01 & 1,02 \\
\hline Mínimo & & 4,00 & 3,00 & 3,00 & 2,00 \\
\hline Máximo & & 14,00 & 12,00 & 12,00 & 7,00 \\
\hline
\end{tabular}

recurso à validade externa pode constituir uma limitação, ainda que este tipo de procedimento possa ser implementado em estudos futuros que se realizem com a EAMC, no sentido de analisar a robustez psicométrica da mesma.

Destaca-se que a grande maioria dos estudantes acredita ser pertinente a promoção de alguma forma de apoio relativamente a situações que possam desencadear maior grau de ansiedade, o que poderá alertar para a pertinência da dimensão em causa e a sua avaliação.

Como principais limitações deste estudo, apontam-se o efeito da desejabilidade social, inerente a qualquer auto-questionário, e o facto de a amostra em estudo ser uma amostra por conveniência, condicionando a generalização dos resultados. Além disso, não foi feito um teste-reteste, o que permitiria a análise da estabilidade da variável em estudo.

Desta forma consideramos importante a sua aplicação em estudos futuros, o que permitirá aprofundar o estudo das características psicométricas da escala e dotar o instrumento de maior garantia de estabilidade. Reforçamos a pertinência de recorrer futuramente ao estudo da validade externa, através, por exemplo, da validação cruzada e correlação com outras variáveis.

\section{REFERÊNCIAS}

1. Bayram N, Bilgel, N. The prevalence and sociodemographic correlations of depression, anxiety and stress among a group of university students. Soc Psychiatr Epidemiol. 2008;43:667-2.

2. Kötter T, Tautphäus $Y$, Scherer M, Voltmer E. Health-promoting factors in medical students and students of science, technology, engineering, and mathematics: design and baseline results of a comparative longitudinal study. BMC Med Educ. 2014;14:134.

3. Dyrbye L, Thomas M, Shanafelt T. Systematic review of depression, anxiety, and other indicators of psychological distress among U.S. and Canadian medical students. Acad Med. 2006;81:354-73.

4. Dicionário de termos médicos. $2^{\mathrm{a}}$ ed. Porto: Porto Editora; 2005.

5. Dicionário Médico Enciclopédico - Taber. $17^{\mathrm{a}}$ ed. Lisboa: Lusodidacta Limitada; 2000.

6. Crujo M, Marques C. As perturbações emocionais - ansiedade e depressão na criança e no adolescente. Rev Port Clinic Geral. 2009;25:576-82.

\section{CONCLUSÃO}

A EAMC total demonstra uma boa coerência interna (Alpha de Cronbach $=0,84$ ) e boa capacidade de discriminação de sujeitos apresentando-se assim, como instrumento consistente e fiável para a avaliação de ansiedade nos estudantes de Medicina quando expostos ao meio clínico. Desta forma, a aplicação desta escala poderá vir a ser útil na delineação de estratégias de ensino para a preparação dos futuros médicos. A quase totalidade dos estudantes considerou pertinente a promoção, pelos estabelecimentos de ensino de alguma forma de apoio relativamente a situações que possam desencadear maior grau de ansiedade em meio clínico. Tais atividades poderão, assim, representar um benefício para muitos estudantes, apesar da baixa prevalência de ansiedade em meio clínico constatada neste estudo.

\section{CONFLITOS DE INTERESSE}

Os autores declaram não ter qualquer conflito de interesse relativamente ao presente artigo.

\section{FONTES DE FINANCIAMENTO}

Este trabalho não recebeu qualquer contribuição e subsídio ou bolsa.

7. Cunha M, Neves A, Moreira M, Henh F, Lopes T, Ribeiro C, et al. Transtornos psiquiátricos menores e procura por cuidados em estudantes de medicina. Rev Bras Educ Med. 2009;33:321-8.

8. Aguiar S, Vieira A, Vieira K, Aguiar S, Nobrega J. Prevalência de sintomas de estresse nos estudantes de medicina. J Bras Psiquiatr. 2009;58:34-38.

9. Frasquilho M. Medicina, uma jornada de 24 horas? Stress e burnout em médicos: prevenção e tratamento. Rev Port Saude Publica. 2005;23:8998.

10. Dyrbye L, West C, Satele D, Boone S, Tan L, Sloan J, et al. Burnout among U.S. medical students, residents, and early career physicians relative to the general U.S. population. Acad Med. 2014;89:443-51.

11. Ritter H. Anxiety. J Religion Health. 1990;29:49-53.

12. Ramos F. El Síndrome de burnout. Madrid: UNED-FUE; 1999.

13. Lima M, Domingues $M$, Cerqueira A. Prevalência e fatores de risco para 
transtornos mentais comuns entre estudantes de medicina. Rev Port Saude Publica. 2006;40:1035-41.

14. Chandavarkar $U$, Azzam A, Mathews $C$. Anxiety symptoms and perceived performance in medical students. Depress Anxiety. 2007;24:10311.

15. Peixoto B, Saraiva C, Sampaio D. Comportamentos suicidários em Por- tugal. Coimbra: Sociedade Portuguesa de Suicidologia; 2006.

16. Maroco J. Análise estatística com utilização do SPSS. $3^{a}$ ed. Lisboa: Edições Sílabo; 2007

17. DeVellis R. Scale development: theory and applications. Newbury Prak: Sage Publications; 1991. 


\section{Ansiedade em Meio Clínico: Construção de uma Escala para Estudantes de Medicina}

Acta Med Port 2014:27:731-736

Publicado pela Acta Médica Portuguesa, a Revista Científica da Ordem dos Médicos

Av. Almirante Gago Coutinho, 151

1749-084 Lisboa, Portugal.

Tel: +351218428215

E-mail: submissao@actamedicaportuguesa.com

www.actamedicaportuguesa.com

ISSN:0870-399X | e-ISSN: 1646-0758

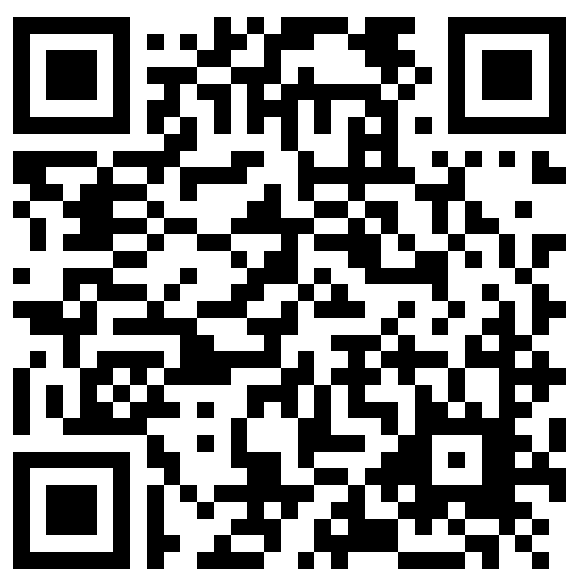

